\title{
Is there a subgroup of long-term evolution among patients with advanced lung cancer?: Hints from the analysis of survival curves from cancer registry data
}

\author{
Lizet Sanchez ${ }^{1 *}$, Patricia Lorenzo-Luaces ${ }^{1}$, Carmen Viada ${ }^{1}$, Yaima Galan², Javier Ballesteros ${ }^{3}$, Tania Crombet $^{4}$ \\ and Agustin Lage 5* $^{*}$
}

\begin{abstract}
Background: Recently, with the access of low toxicity biological and targeted therapies, evidence of the existence of a long-term survival subpopulation of cancer patients is appearing. We have studied an unselected population with advanced lung cancer to look for evidence of multimodality in survival distribution, and estimate the proportion of long-term survivors.

Methods: We used survival data of 4944 patients with non-small-cell lung cancer (NSCLC) stages IIIb-IV at diagnostic, registered in the National Cancer Registry of Cuba (NCRC) between January 1998 and December 2006. We fitted one-component survival model and two-component mixture models to identify short- and long- term survivors. Bayesian information criterion was used for model selection.

Results: For all of the selected parametric distributions the two components model presented the best fit. The population with short-term survival (almost 4 months median survival) represented $64 \%$ of patients. The population of long-term survival included 35\% of patients, and showed a median survival around 12 months. None of the patients of short-term survival was still alive at month 24 , while $10 \%$ of the patients of long-term survival died afterwards.
\end{abstract}

Conclusions: There is a subgroup showing long-term evolution among patients with advanced lung cancer. As survival rates continue to improve with the new generation of therapies, prognostic models considering short- and long-term survival subpopulations should be considered in clinical research.

Keywords: Long-term survivors, Survival, Mixture models, Non-small-cell lung cancer

\section{Background}

For decades, the primary focus of cancer research was the development of therapeutic interventions to cure the cancer or produce a remission. Success with standard cancer therapy (surgery, radiotherapy and chemotherapy combinations) was mainly limited to early stage tumors. Because of the natural history of cancer, it is relevant to understand if we are witnessing real cures, or just delays

\footnotetext{
*Correspondence: Isanchez@cim.sld.cu; lage@cim.sld.cu

${ }^{1}$ Clinical Research Division, Center of Molecular Immunology, Calle 216 esq 15, Atabey, Havana 11600, Cuba

${ }^{5}$ Center of Molecular Immunology, Calle 216 esq 15, Atabey, Havana 11600, Cuba

Full list of author information is available at the end of the article
}

in the transition to advanced disease at a given rate [1]. Survival analysis addresses such issues.

The relative survival curve for many cancers will reach a plateau some years after diagnosis, indicating that the mortality among patients still alive at that point is near to the expected mortality in the general population [2]. A straightforward way to identify whether a particular dataset might include a subset of long-term survivors is thus to look at the survival curve to identify the existence or not of such plateau [3]. Another approach is to perform a visual inspection of the hazard function (instantaneous risk of death) plot to look for temporal changes suggesting a "cure" might have been achieved for some patients [4]. 
In most analyses of cancer survival data, the main outcomes (overall survival and/or progression-free survival) are estimated from conventional methods as Kaplan-Meier and Cox regression models. However, these methods might fail to describe adequately the heterogeneity among cancer patients [5]. To overcome that drawback Boag [6] proposed a two-component mixture model for the analysis of survival data when it is known that a proportion of patients are cured. Such cure models, explicitly model survival as a mixture of cured patients (usually modeled using logistic regression approaches) and non-cured patients (usually modeled using survival approaches).

Many variations of cure models have been proposed and extensively applied. However, the applications have been mainly for patients diagnosed at early stages of cancer [7-11]. Almost all reports have used simulated data or have applied the different models to breast or colon cancer in curable stages.

Exploration of survival data looking for a "cured fraction" has not been extensively applied for advanced cancer, where clinical experience indicates that "cures" are extremely rare or even do not exist. Particularly in lung cancer, without curative treatments for patients in advanced stages, few studies have reported applications of mixture cure models [12].

Recently, and because the advent of biological therapies presenting low toxicity, and targeted therapies, evidences of the existence of a long-term survival subpopulation of patients are beginning to appear, and it is thus relevant to know if this subpopulation represents the tail of the survival distribution that have been shifted towards longer survival by the therapy being administered, or if it represents the existence of intrinsic heterogeneity in the patient population, causing multimodality in the distribution of survival times. If such a chronic evolution subpopulation exists, even in the advanced cancer situation, and some patients live enough to allow the intervention of competing causes of death, it could be convenient to think in terms of long-term survivors or "statistically" cured patients [13].

Finally, it should be noted that the presence of multimodality or mixture distributions in cancer patients could be obscured when clinical trials are the main data source for the analysis, because patients included in clinical trials are by definition selected for reduction of heterogeneity.

In the present paper several parametric survival models and mixture models were applied to an unselected population of patients with advanced lung cancer to look for evidence of multimodality in the survival distribution, and to estimate the proportion of long-term survivors.

\section{Methods}

Data

The NCRC registers all cancers diagnosed in Cuba [14]. Information within cancer registrations is ascertained from hospital records, diagnostic procedures, pathology reports and death certificates. The estimate of registration completeness at NCRC is $80 \%$ [15]. Incident cases of NSCLC reported by NCRC were linked to death records provided by the Cuban National Statistics Office of the Ministry of Public Health.

All adults over 18 years, diagnosed with histological or cytological proven non-small-cell lung cancer (NSCLC) at stages IIIb or IV between January 1998 and December 2006, who were registered in the National Cancer Registry of Cuba (NCRC) with follow-up to December 31, 2010 were eligible for analysis. Of the 6425 eligible patients, 4944 (76.9\%) were linked with death records using personal identification number. Due to missing or incorrect identification, $11.2 \%$ of patients were excluded from the analysis. The rest of the patients $(11.9 \%)$ were classified as loss of follow up and were also excluded.

\section{Modeling approach}

For the one component model, the survival function $\mathrm{S}(\mathrm{t})$ for the overall population survival time and the hazard, the instantaneous risk of death, were fitted assuming the following parametric models: Gaussian, Log-normal, Weibull and Gamma. Additionally, we fitted a two-component mixture model considering the same distributions adjusted to identify short- and long- term survivors within the advanced lung cancer patients. The survival function for overall population survival time $\mathrm{T}$ was expressed as:

$$
\mathrm{S}(\mathrm{t})=\mathrm{c}_{1} \mathrm{G}\left(\mathrm{t} \mid \mu_{1}, \sigma_{1}\right)+\mathrm{c}_{2} \mathrm{G}\left(\mathrm{t} \mid \mu_{2}, \sigma_{2}\right)
$$

Where $\mathrm{G}(\mathrm{t} \mid \mu, \sigma)$ is a distribution function. The parameters $c_{k},(k=1,2)$, with the restriction that $0<c_{1}<c_{2} \leq 1$ and $c_{1}+c_{2}=1$, are the mixed fractions for the $K$ population. The fractions $\mathrm{c} 1$ and $\mathrm{c} 2$ can be interpreted as the proportion of short-term and long-term survivors respectively. In the model $\left(\mu_{\mathrm{k}}, \sigma_{\mathrm{k}}\right)$, are the parameters of the parametric distribution $\mathrm{G}$.

The maximum likelihood estimators of the parameters $(c, \mu, \sigma)$ for the one component or two component mixture models were found by maximizing the likelihood function. We used R v3.0.2 (R Core Team, 2013) for the statistical analyses with the EM algorithm implemented in the "rebmix" library [15] of R (R software; http://www.rproject.org).

\section{Model selection}

We compared the parametric models with the Bayesian information criterion $\left(\mathrm{BIC}=-\log (\right.$ likelihod $)+\frac{p}{2} \log (n)$, where $\mathrm{p}$ is the number of parameters and $\mathrm{n}$ is the sample size) to find the most probable model given the data. The model with the smallest BIC value was considered the best fit to the observed data. A BIC difference $>10$ between the more complex model assuming two components and the 
simplest model with only one component was considered as very strong evidence to support the two components approach against the simplest alternative [16].

\section{Ethics}

The use of the data here reported was approved for research purposes by the appropriate Ethical and Research Commitee of the National Cancer Registry of Cuba. Anonymized records (non-patient identifiable data) were provided by the NCRC.

\section{Results}

The median survival time of the Cuban advanced NSCLC patients was 3.93 months. Note that in the survival curve (Figure 1a) it is possible to distinguish a plateau at the end of the study period. Accordingly, the hazard function (Figure 1b) shows a monotonic decreasing curve. Both graphics suggest the presence of two different populations.

For all of the selected parametric distributions (Gaussian, log-normal, Weibull or Gamma), the two components model presented the best fit. Gaussian distribution showed the greatest changes in BIC values, while the Gamma distribution provided the best fit to the data (see Table 1). In all models the BIC difference between one- and twocomponent models was greater than 10 , supporting the most complex model and thus the likely existence of two populations of patients. In the Gamma model, the population with short term survival (almost 4 months median survival) represented $64 \%$ of NSCLC patients. The population of long-term survivors, which included $35 \%$ of patients, showed a median survival close to 12 months.

Models assuming Gaussian and Gamma distributions were selected to illustrate the density and cumulative survival curves for short-term and long-term survival populations (Figure 2). Figure 2a and d show the density functions for Gaussian and Gamma distribution respectively. The density peak at 4 months for the first population, indicates that most patients died at that moment. However in the second population the density is flattened. Figure $2 \mathrm{~b}$ shows no survivors after 11 month for short-term survival population whereas $45 \%$ of longterm survival population is still alive. Nevertheless, assuming Gamma distribution (Figure 2e), no patients of the first population are still surviving at month 24, while $10 \%$ of long-term survival population died afterwards. As seen, the mixture curves, either for Gaussian or for Gamma distributions, fit quite well the observed survival (Figure 2c, f).

\section{Discussion}

Is there a subgroup with long-term survival among patients with advanced lung cancer? Our data suggest an affirmative answer. The survival data of advanced NSCLC patients reported by the NCRC could be best explained by
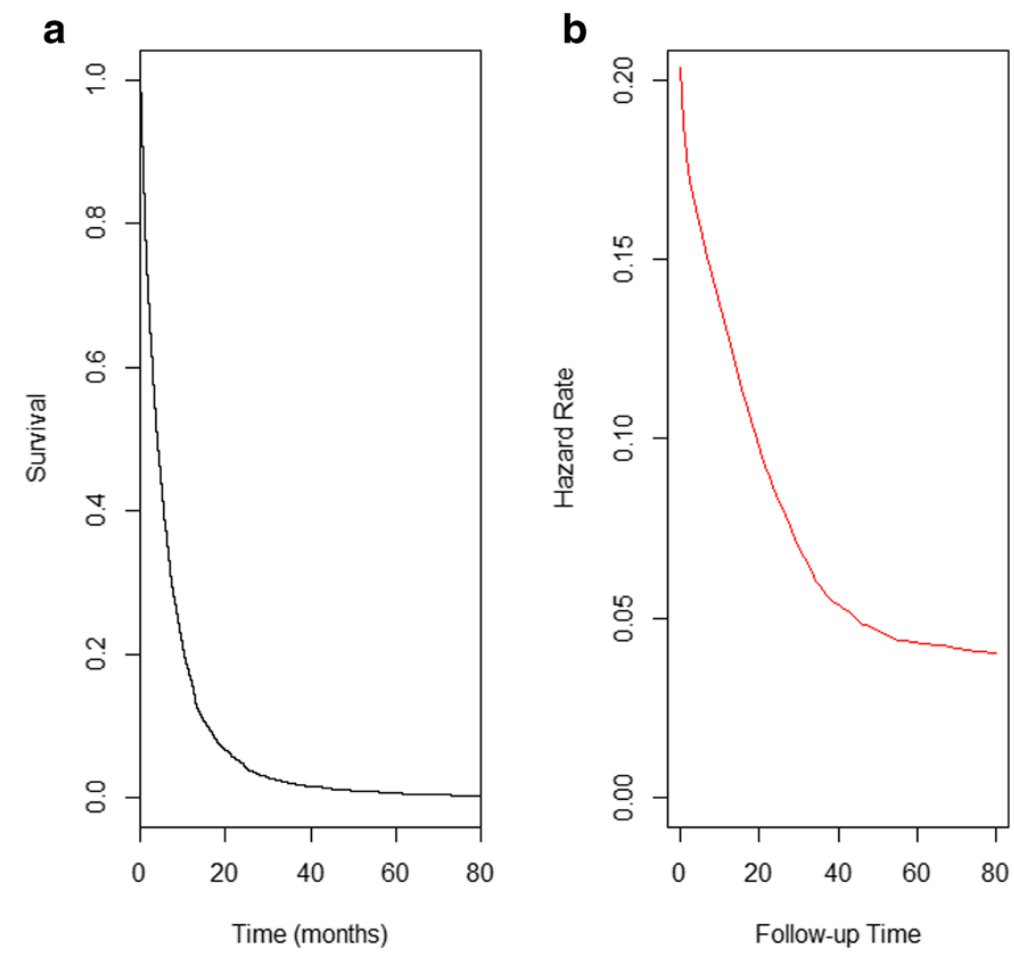

Figure 1 Cumulative survival a) and hazard curves b) for advanced non-small cell lung cancer registry by the Cuban Cancer National Registry. 1998-2006. 
Table 1 Mix fraction and median survival times estimated for short- and long- term survival populations using different parametric models

\begin{tabular}{|c|c|c|c|c|c|c|}
\hline \multirow[t]{2}{*}{ Distribution } & \multirow[t]{2}{*}{$\begin{array}{l}\text { Numbers of } \\
\text { components } \\
\text { in the model }\end{array}$} & \multicolumn{2}{|c|}{$\begin{array}{l}\text { Short term } \\
\text { survival } \\
\text { population }\end{array}$} & \multicolumn{2}{|c|}{$\begin{array}{l}\text { Long term } \\
\text { survival } \\
\text { population }\end{array}$} & \multirow[t]{2}{*}{ BIC } \\
\hline & & c & Median & c & Median & \\
\hline \multirow[t]{2}{*}{ Gaussian } & One & 1 & 7.21 & - & & 36622.4 \\
\hline & Two & 0.80 & 3.86 & 0.20 & 19.9 & 31353.9 \\
\hline \multirow[t]{2}{*}{ Weibull } & One & 1 & 8.27 & - & & 30320.8 \\
\hline & Two & 0.92 & 9.17 & 0.08 & 10.10 & 29528.7 \\
\hline \multirow[t]{2}{*}{ Log normal } & One & 1 & 4.52 & - & & 29094.0 \\
\hline & Two & 0.77 & 4.22 & 0.23 & 6.57 & 28942.5 \\
\hline \multirow[t]{2}{*}{ Gamma } & One & 1 & 7.25 & - & & 29250.5 \\
\hline & Two & 0.64 & 3.57 & 0.35 & 11.9 & 28610.3 \\
\hline
\end{tabular}

c, Mix fraction in the total population; BIC, Bayesian information criterion. The model with the smallest value of BIC has the best fit. a complex mixture model of two populations than for a simpler model assuming only one homogeneous population. In summary, the results provides evidence of the existence of a mixture of populations, including one with long-term survival, consisting of more than $10 \%$ of all reported cases, with a survival time greater than 24 months.

Therapies for certain cancer types are believed to induce a subset of long term survivors, such as melanoma [17], breast cancer [18] and multiple myeloma [3]. On the other hand, population based studies have reported the cure fraction estimates for breast $[5,12,19]$ and colorectal cancer $[13,20]$. However, to our knowledge, this is the first study in an unselected population with advanced NSCLC patients that has found compelling evidence of the existence of a subgroup of patients presenting longterm evolution.

In spite of the fitting complexity of the mixture model, its parameters have a very intuitive interpretation for clinicians. Each subpopulation can be distinguished by two attributes: its size or mix fraction, expressed in percentage; and the corresponding median survival time. It is
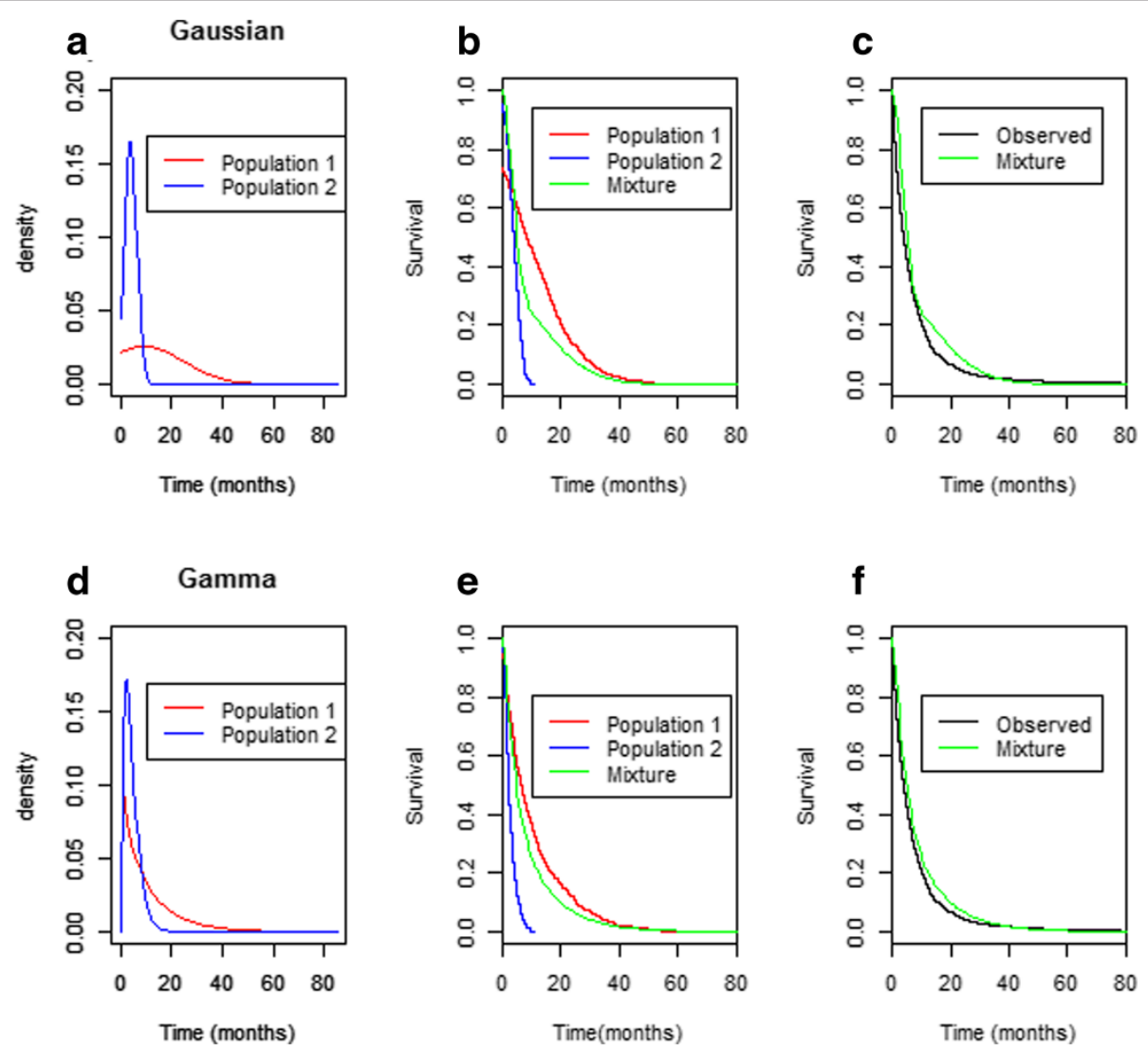

Figure 2 Illustration of survival patterns of short-term, long-term and mixture populations. a) Density survival curves assuming Gaussian distribution b) Cumulative survival curves for short-term, long-term and mixture assuming Gaussian distribution c) Observed vs estimated overall survival assuming mixture of two Gaussian distributions d) Density survival curves assuming Gamma distribution e) Cumulative survival curves for short-term, long-term and mixture assuming Gamma distribution f) Observed vs estimated overall survival assuming mixture of two Gamma distributions. 
important to note that estimates of mix fraction can be very sensitive to the parametric distribution chosen to work with. Sometimes, the distribution may not be flexible enough to capture the overall shape of the survival distribution [13]. For this reason, the selection of the parametric distribution to model the observed data should be done carefully. McCullagh and Barry [21] proposed a model selection process algorithm and recommended to fit different distributions to the data to select the best one by using one of the available information criteria.

There are some limitations to both the data and the methodology used in this study. The completeness of NCRC data is known to be high, but may be biased by uncorrected diagnosis dates. Some studies have found this issue to have minimal impact on survival [22]. Stage-specific cure has rarely been estimated due the large proportion of cancer without code of stage in population-based data. Another possible source of bias is that patients without death certificate were excluded from the analysis. As a consequence, under-estimation of survival rates could have happened. However, studies aims to measure that bias, concluded that the effect is minimal when data from population-based cancer registry is used, indicating that the losses can be considered practically random [23,24]. Furthermore, $\mathrm{Yu}$ [20] emphasizes that mixture cure models should be used when there is sufficient follow-up beyond the time when most events occurs. In the case of advanced NSCLC, although estimated median survivals are in the range of 8 to 10 months, several reports [25-27] support the existence of long term survivors - defined as those surviving for more than 2 years after a diagnosis of extensive NSCLC [28].

The transition of advanced cancer to chronicity is a concept that has recently emerged in the literature. Research in cancer treatment has been focused on the search for "cures", in a naïve extrapolation of the success of antibiotics against infections. This therapeutic paradigm is currently in change driven by the success of modern treatments in prolonging survival in patients with advanced cancer with an ethically acceptable quality of life [29-31], and thus research focus is also moving towards the long term control of the advanced disease. As an analogy worth to note, the history of therapeutic research in Type 1 Diabetes run exactly in the opposite way: whereas it started looking for long term control, and remained so for decades, the therapeutic shift to its "cure" has only become a focus of attention, through the current experimental technologies of pancreatic islet transplants.

Despite their theoretical appearance, these intellectual frames can have huge practical implications for the way clinical research is designed and analyzed. The importance of accounting for long term survivors when the efficacy and safety of immune-oncologic agents is evaluated has been highlighted before [32]. The log rank test and Cox regression models, the standard analyses in immunotherapy evaluation, have maximal statistical power under the proportional hazard assumption. However, Cox models can only provide a satisfactory description of relative survival of the various population groups in the early years after treatment begins, as they cannot present a plateau. Moreover, as survival rates continue to improve, long term survival and cure are becoming increasingly important endpoints when planning oncological clinical trials.

\section{Further research}

Further research is needed to explore the effect of individual prognostic factors and the effect of treatments on the proportion and the failure time of long-term and short-term survival patients. Few current clinical trials have been designed and consequently analyzed with that perspective. Systematic analysis of heterogeneity in survival curves, and of the impact of treatments, not just in the attributes of the survival curves, but on the internal distribution of survival subpopulations, could provide novel and fertile avenues of research.

\section{Conclusions}

This study analysed the survival distribution of advanced NSCLC patients registered in the NCRC. It provides evidence of the existence of a mixture of populations, including a subgroup showing long-term evolution. As survival rates continue to improve with the new generation of therapies, prognostic models considering shortand long- term survival subpopulation should be considered in clinical research. Be able to increase the proportion of patients in the long- term survival group could be a desirable goal for cancer control programs.

\section{Competing interests}

We declare that we don't have any competing interests to declare in relation to this manuscript

\section{Authors' contributions}

$\mathrm{LS}, \mathrm{PL}$ and $\mathrm{AL}$ conceived the study, participated in data analysis, and drafted the manuscript. YG participated in the data collection and quality control of data from the National Cancer Registry. CV, TC and JB participated in data analysis and drafted the manuscript. All authors participated in the interpretation of the data and critically revised subsequent drafts of the manuscript. All authors read and approved the final manuscript.

\section{Acknowledgements}

$\mathrm{LS}, \mathrm{PL}, \mathrm{CV}, \mathrm{TC}, \mathrm{AL}$ were funded by their employer the Center of Molecular Immunology. YG is funded by the Ministry of Health. JB received no funding. We thank Dr. Camilo Rodriguez for their contribution to this work and for facilitate literature needed for manuscript writing.

\section{Author details}

${ }^{1}$ Clinical Research Division, Center of Molecular Immunology, Calle 216 esq 15, Atabey, Havana 11600, Cuba. ${ }^{2}$ National Cancer Registry, 29 y F, vedado, Havana 10400, CUBA. ${ }^{3}$ University of the Basque Country, UPV/EHU, and CIBERSAM, Barrio Sarriena s/n, Leioa 48940, Spain. ${ }^{4}$ Clinical Research Direction, Center of Molecular Immunology, Calle 216 esq 15, Atabey, Havana 11600, Cuba. ${ }^{5}$ Center of Molecular Immunology, Calle 216 esq 15, Atabey, Havana 11600, Cuba. 
Received: 19 April 2014 Accepted: 20 November 2014

Published: 11 December 2014

\section{References}

1. Lage A, Pascual MR, Pérez R: Estudios sobre el pronóstico del cáncer mamario. Análisis de las curvas de mortalidad y recaída en el cáncer de mama. Rev Cub Oncol 1986, 2:21-29.

2. Andersson TM, Dickman PW, Eloranta S, Lambert PC: Estimating and modelling cure in population-based cancer studies within the framework of flexible parametric survival models. BMC Med Res Methodol 2011, 11:96.

3. Othus M, Barlogie B, Leblanc ML, Crowley JJ: Cure models as a useful statistical tool for analyzing survival. Clin Cancer Res 2012, 18(14):3731-3736.

4. Weston CL, Douglas C, Craft AW, Lewis IJ, Machin D: Establishing longterm survival and cure in young patients with Ewing's sarcoma. $\mathrm{Br} J$ Cancer 2004, 91(2):225-232.

5. Yilmaz YE, Lawless JF, Andrulis IL, Bull SB: Insights from mixture cure modeling of molecular markers for prognosis in breast cancer. J Clin Oncol 2013, 31(16):2047-2054.

6. Boag JM: Maximum likelihood estimates of the proportion of patients cured by cancer therapy. J R Stat Soc B 1949, 11:15-44.

7. Chen WC, Hill BM, Greenhouse JB, Fayos JV: Bayesian analysis of survival curves for cancer patients following treatment. Bayesian Stat 1985, 2:299-328.

8. Maller RA, Zhou S: Testing for sufficient follow-up and outliers in survival data. J Am Stat Assoc 1994, 89:1499-1506.

9. Angelis R, Capocaccia R, Hakulinen T, Soderman B, Verdecchia A: Mixture models for cancer survical analysis: aplication to population-based data with covariates. Stat Med 1999, 18:144-454.

10. Zhan J, Peng Y: Accelerated hazards mixture cure model. Lifetime Data Anal 2009, 15:455-467.

11. Marin JM, Rodriguez-Bernal MT, Wiper MP: Using weibull mixture distributions to model heterogeneous survival data. Communicat Stat 2005, 34:673-684.

12. Yu B, Tiwari RC, Cronin KA, Feuer EJ: Cure fraction estimation from the mixture cure models for grouped survival data. Stat Med 2004, 23(11):1733-1747.

13. Lambert PC, Thompson JR, Weston CL, Dickman PW: Estimating and modeling the cure fraction in population-based cancer survival analysis. Biostatistics 2007, 8(3):576-594.

14. Galan Y, Fernandez L, Torres P, Garcia M: Trends in Cuba's Cancer Incidence (1990 to 2003) and mortality (1990 to 2007). MEDICC Rev 2009, 11(3):19-26

15. Nagode M, Fajdiga M: The REBMIX algorithm for the univariate finite mixture estimation. Communicat Stat 2011, 40(5):876-892.

16. Wasserman L: Bayesian model selection and model averaging. $J$ Math Psychol 2000, 44(1):92-107.

17. Eggermont AM, Suciu S, Testori A, Santinami M, Kruit WH, Marsden J, Punt CJ, Sales F, Dummer R, Robert C, Schadendorf D, Patel PM, de Schaetzen G, Spatz A, Keilholz U: Long-term results of the randomized phase III trial EORTC 18991 of adjuvant therapy with pegylated interferon alfa-2b versus observation in resected stage III melanoma. J Clin Oncol 2012, 30(31):3810-3818.

18. Ambs S: Prognostic significance of subtype classification for short- and long-term survival in breast cancer: survival time holds the key. PLoS Med 2010, 7(5):e1000281.

19. Zhao Y, Lee AH, Yau KK, Burke V, McLachlan GJ: A score test for assessing the cured proportion in the long-term survivor mixture model. Stat Med 2009, 28(27):3454-3466.

20. Yu B, Tiwari RC: Application of EM algorithm to mixture cure model for grouped relative survival data. J Data Sci 2007, 5:10.

21. McCullagh L, Barry M: Survival analysis used in company submissions to the national centre for pharmacoeconomics. Ireland Value Health 2013, 16:A398.

22. Shack LG, Shah A, Lambert PC, Rachet B: Cure by age and stage at diagnosis for colorectal cancer patients in North West England, 19972004: a population-based study. Cancer Epidemiol 2012, 36(6):548-553.

23. Swaminathan $R$, Rama R, Shanta V: Lack of active follow-up of cancer patients in Chennai, India: implications for population-based survival estimates. Bull World Health Organ 2008, 86(7):509-515.
24. Dhar M, Rao S, Vijaysimha R: Population based studies of cancer survival: scope for the developing countries. Asian Pac J Cancer Prev 2010, 11(3):831-838.

25. Wang T, Nelson RA, Bogardus A, Grannis FW Jr: Five-year lung cancer survival: which advanced stage nonsmall cell lung cancer patients attain long-term survival? Cancer 2010, 116(6):1518-1525.

26. Ahbeddou N, Fetohi M, Boutayeb S, Errihani H: Which non-small-cell lung cancer patients achieve long-term survival? Indian J Cancer 2011, 48(4):514-515.

27. Ozkaya S, Findik S, Dirican A, Atici AG: Long-term survival rates of patients with stage IIIB and IV non-small cell lung cancer treated with cisplatin plus vinorelbine or gemcitabine. Exp Therap Med 2012, 4(6):1035-1038.

28. Giroux Leprieur E, Lavole A, Ruppert AM, Gounant V, Wislez M, Cadranel J, Milleron B: Factors associated with long-term survival of patients with advanced non-small cell lung cancer. Respirology 2012, 17(1):134-142.

29. Lage A: Connecting immunology research to public health: Cuban biotechnology. Nat Immunol 2008, 9:109-112.

30. Lage A: Transforming cancer indicators begs bold new strategies from biotechnology. MEDICC Rev 2009, 11(3):8-12.

31. Schlom J, Arlen PM, Gulley JL: Cancer vaccines: moving beyond current paradigms. Clin Cancer Res 2007, 13(13):3776-3782.

32. Chen TT: Statistical issues and challenges in immunooncology. J Immuno Ther Cancer 2013, 1:1-9.

doi:10.1186/1471-2407-14-933

Cite this article as: Sanchez et al:: Is there a subgroup of long-term evolution among patients with advanced lung cancer?: Hints from the analysis of survival curves from cancer registry data. BMC Cancer 2014 14:933.

\section{Submit your next manuscript to BioMed Central and take full advantage of:}

- Convenient online submission

- Thorough peer review

- No space constraints or color figure charges

- Immediate publication on acceptance

- Inclusion in PubMed, CAS, Scopus and Google Scholar

- Research which is freely available for redistribution 